\title{
Dieta de Thamnodynastes strigatus (Serpentes, Colubridae) no sul do Brasil
}

\section{Raquel Ruffato, Marcos Di-Bernardo e Gleomar Fabiano Maschio}

Faculdade de Biociências e Museu de Ciências e Tecnologia da Pontifícia Universidade Católica do Rio Grande do Sul, Av. Ipiranga, 6681, 90619-900, Porto Alegre, RS, Brasil. E-mail: madibe@ pucrs.br.

\begin{abstract}
Diet of Thamnodynastes strigatus (Serpentes, Colubridae) in southern Brazil. We analyzed the gut content of 151 specimens of the colubrid snake Thamnodynastes strigatus from the state of Rio Grande do Sul, southern Brazil. The recorded prey items were anurans $(66 \% ; n=33)$, fish $(28,0 \% ; n=14)$, mammals $(4,0 \% ; n=2)$, and reptiles $(2,0 \% ; n=1)$. There was no correlation between the snout-vent length (SVL) of the snakes and the total length (TL) of their prey, but the correlation between the snake SVL and the ratio prey TL / snake SVL was significant and negative. This negative correlation evidences that $T$. strigatus does not exclude proportionally small prey items of their diet, and that, proportionally, the biggest prey items are ingested by the smallest snakes. Relatively large prey items are manipulated before ingestion and oriented headfirst, whereas relatively small prey were swallowed head- or tailfirst.
\end{abstract}

Keywords: Squamata, Serpentes, Colubridae, Thamnodynastes strigatus, diet, southern Brazil.

Palavras-Chave: Serpentes, Colubridae, Thamnodynastes strigatus, dieta, sul do Brasil.

\section{Introdução}

As serpentes do gênero Thamnodynastes Wagler, 1830 têm distribuição ampla na América do Sul; mesmo assim, há poucas informações sobre biologia, morfologia ou taxonomia (Franco 1999, Franco e Ferreira 2002). As espécies são predominantemente noturnas (Strüssmann e Sazima 1993, Marques 1998, Lema 2002) e apresentam tamanho pequeno a médio, sendo Thamnodynastes strigatus (Günther 1858) a de maior porte (Cei 1993,

Recebido em 13 de junho de 2003

Aceito em 8 de agosto de 2003

Distribuído em 31 de outubro de 2003
Giraudo 2001, Franco e Ferreira 2002). Embora vários autores tenham relatado a inconsistência da situação taxonômica das espécies de Thamnodynastes (e.g. Bailey 1967, Vanzolini et al. 1980, Franco e Ferreira 2002, R. A. Thomas, com. pess.), Franco (1999) e Franco e Ferreira (2002) apresentaram diagnose e descrição suficientes para o reconhecimento de $T$. strigatus.

Thamnodynastes strigatus é um colubrídeo opistóglifo de hábito noturno (Abuys 1986, Bizerra 1998, Bernarde et al. 2000b, Marques et al. 2001) pertencente à tribo Tachymenini (Bailey 1967). Ocorre no Paraguai, Argentina, sul e sudeste do Brasil e Uruguai (Peters e Orejas-Miranda 1970, Lema et al. 1984, 
Achaval-Elena 2001, Franco e Ferreira 2002, Lema 2002). No Brasil, os registros para a espécie estão inseridos em áreas do Domínio Tropical Atlântico e das Coxilhas (Ab'Saber 1977, Franco e Ferreira 2002). Apesar de ser uma espécie relativamente comum, sua biologia e ecologia são pouco conhecidas. Amaral (1978) e Achaval e Olmos (1997) citaram como itens alimentares da espécie apenas lagartos e pequenos roedores, mas Franco (1999) considerou $T$. strigatus uma serpente generalista (conforme Schoener 1971). Cechin (1999) encontrou anuros como suas principais presas e mamíferos como presas ocasionais. Bernarde et al. (2000a, b) consideraram T. strigatus uma espécie anurófaga, que inclui ocasionalmente roedores, peixes e lagartos na dieta.

Neste estudo, apresentamos a composição da dieta e a freqüência relativa das categorias de presas consumidas por espécimes de $T$. strigatus procedentes do sul do Brasil.

\section{Material e Métodos}

A composição e a frequiência relativa dos itens alimentares foram obtidos pela análise do conteúdo do tubo digestivo (estômago e intestino) de 151 espécimes de $T$. strigatus procedentes do estado do Rio Grande do Sul, depositados nas coleções do Museu de Ciências e Tecnologia da Pontifícia Universidade Católica do Rio Grande do Sul (MCP), Museu de Ciências Naturais da Fundação Zoobotânica do Rio Grande do Sul (MCN), Setor de Zoologia da Universidade Federal de Santa Maria (ZUFSM) e Coleção de Répteis da Universidade de Passo Fundo (CRUPF). Espécimes alimentados em cativeiro não foram utilizados na análise. Os itens alimentares foram identificados e depositados nas coleções acima citadas.

$\mathrm{Na}$ análise da relação de tamanho entre predador (serpente) e presa (item alimentar), o tamanho do predador refere-se ao comprimento rostro-cloacal (CRC), medido indiretamente estendendo-se um barbante ao longo da superfície ventral de cada indivíduo, desde a tangente à extremidade do focinho até o centro da escama cloacal; em seguida, o barbante foi medido com régua milimetrada com precisão de $1 \mathrm{~mm}$. $\mathrm{O}$ tamanho da presa refere-se ao comprimento total (CT) e foi medido com paquímetro com precisão de $0,01 \mathrm{~mm}$. Presas intactas ou pouco digeridas foram medidas diretamente. Em alguns casos, partes anatômicas íntegras de presas muito digeridas foram medidas e usadas para inferir seu CT por meio da comparação de tamanho com presas da mesma espécie depositadas em coleções. O sentido de ingestão das presas foi determinado observando-se a posição relativa de cada presa no interior do tubo digestivo das serpentes, sendo indicado como ântero-posterior, para presas ingeridas a partir da região anterior do corpo, e póstero-anterior, para presas ingeridas a partir da região posterior do corpo. Presas muito digeridas ou em posição transversal no tubo digestivo não foram incluídas nessa análise.

As correlações tamanho presa / predador foram analisadas empregando-se o Teste de Correlação Linear de Pearson.

\section{Resultados}

Dos 151 espécimes de Thamnodynastes strigatus analisados, $28(18,5 \%)$ apresentaram 50 itens alimentares no estômago ou intestino. A dieta foi composta por anfíbios anuros $(\mathrm{n}=$ 33 [66,0\%]; 30 ocorrências [81,1\%] em 22 serpentes), peixes ( $n=14$ [28,0\%]; quatro ocorrências $[10,8 \%]$ em três serpentes), mamíferos (n $=2$ [4,0\%]; duas ocorrências [5,4\%] em duas serpentes) e répteis $(\mathrm{n}=1$ [2,0\%]; uma ocorrência $[2,7 \%]$ em uma serpente) (Tabela 1$)$. Foi possível verificar o sentido de ingestão para 38 itens alimentares, sendo 35 (92,1\%) ântero-posterior e três $(7,9 \%)$ póstero-anterior (Tabela 2$)$. A maioria dos exemplares $(\mathrm{n}=25 ; 89,3 \%)$ continha somente um item alimentar no tubo digestivo. Dois espécimes $(7,1 \%)$ continham seis e sete itens, todos anuros, e um espécime $(3,6 \%)$ continha 12 itens, todos peixes da família Characidae. 
Tabela 1 - Tipos de presas e sua ocorrência na dieta de 28 espécimes de Thamnodynastes strigatus do sul do Brasil. O total do " $n$ " na primeira coluna $(n=37)$ indica que algumas serpentes ingeriram mais de um tipo de presa (veja texto).

\begin{tabular}{|c|c|c|c|c|}
\hline & \multicolumn{2}{|c|}{$\begin{array}{c}\text { Serpentes } \\
\text { contendo presas }\end{array}$} & \multicolumn{2}{|c|}{$\begin{array}{c}\text { Quantidade } \\
\text { de presas }\end{array}$} \\
\hline & $\mathbf{n}$ & $\%$ & $\mathbf{n}$ & $\%$ \\
\hline \multicolumn{5}{|l|}{ Anfíbios (Anura) } \\
\hline \multicolumn{5}{|l|}{ LEPTODACTYLIDAE } \\
\hline Leptodactylus ocellatus & 3 & 8,1 & 4 & 8,0 \\
\hline Leptodactylus gracilis & 1 & 2,7 & 1 & 2,0 \\
\hline Odontophrynus americanus & 4 & 10,8 & 5 & 10,0 \\
\hline Physalaemus sp. & 1 & 2,7 & 1 & 2,0 \\
\hline Não identificado & 5 & 13,5 & 5 & 10,0 \\
\hline \multicolumn{5}{|l|}{ HYLIDAE } \\
\hline Scinax fuscovarius & 1 & 2,7 & 1 & 2,0 \\
\hline Scinax sp. & 2 & 5,4 & 3 & 6,0 \\
\hline Hyla pulchella & 1 & 2,7 & 1 & 2,0 \\
\hline Hyla sp. & 1 & 2,7 & 1 & 2,0 \\
\hline Não identificado & 3 & 8,1 & 3 & 6,0 \\
\hline \multicolumn{5}{|l|}{ BUFONIDAE } \\
\hline Bufo ictericus & 1 & 2,7 & 1 & 2,0 \\
\hline Anuros não identificados & 7 & 19,0 & 7 & 14,0 \\
\hline \multicolumn{5}{|l|}{ Peixes } \\
\hline \multicolumn{5}{|l|}{ CHARACIDAE } \\
\hline Escamas & 1 & 2,7 & 1 & 2,0 \\
\hline Tetragonopterinae & 1 & 2,7 & 4 & 8,0 \\
\hline Hyphessobrycon luetkenii & 1 & 2,7 & 8 & 16,0 \\
\hline \multicolumn{5}{|l|}{ POECILIIDAE } \\
\hline Phalloceros caudimaculatus & 1 & 2,7 & 1 & 2,0 \\
\hline \multicolumn{5}{|l|}{ Mamíferos } \\
\hline \multicolumn{5}{|l|}{ CRICETIDAE } \\
\hline Oligoryzomys nigripes & 2 & 5,4 & 2 & 4,0 \\
\hline \multicolumn{5}{|l|}{ Répteis } \\
\hline \multicolumn{5}{|l|}{ COLUBRIDAE } \\
\hline Sibynomorphus ventrimaculatus & 1 & 2,7 & 1 & 2,0 \\
\hline TOTAL & 37 & 100 & 50 & 100 \\
\hline
\end{tabular}


Tabela 2 - Comprimento rostro-cloacal (CRC) dos espécimes analisados de Thamnodynastes strigatus e comprimento total (CT) e sentido de ingestão (SI) de suas presas. Os dados estão apresentados em ordem crescente da razão CT/CRC.

\begin{tabular}{|c|c|c|c|c|}
\hline Presas & CRC (mm) & CT (mm) & CT/CRC & SI \\
\hline Odontophrynus americanus & 640 & 15,21 & 0,02 & Ântero-posterior \\
\hline Odontophrynus americanus & 545 & 16,77 & 0,03 & Póstero-anterior \\
\hline Leptodactylus ocellatus & 545 & 19,74 & 0,04 & Ântero-posterior \\
\hline Physalaemus sp. & 640 & 26,50 & 0,04 & Ântero-posterior \\
\hline Hyla pulchella & 640 & 24,82 & 0,04 & Póstero-anterior \\
\hline Hyla sp. & 640 & 25,38 & 0,04 & Ântero-posterior \\
\hline Leptodactylus ocellatus & 545 & 24,90 & 0,05 & Ântero-posterior \\
\hline Hylidae & 545 & 27,20 & 0,05 & Ântero-posterior \\
\hline Tetragonopterinae & 391 & 23,40 & 0,06 & Ântero-posterior \\
\hline Tetragonopterinae & 391 & 23,40 & 0,06 & Ântero-posterior \\
\hline Tetragonopterinae & 391 & 23,40 & 0,06 & Póstero-anterior \\
\hline Leptodactylus gracilis & 640 & 43,27 & 0,07 & Ântero-posterior \\
\hline Tetragonopterinae & 391 & 26,04 & 0,07 & Ântero-posterior \\
\hline Scinax sp. & 431 & 30,62 & 0,07 & Ântero-posterior \\
\hline Hyphessobrycon luetkenii & 391 & 28,13 & 0,07 & Ântero-posterior \\
\hline Leptodactylidae & 490 & 36,20 & 0,07 & Ântero-posterior \\
\hline Hyphessobrycon luetkenii & 391 & 32,27 & 0,08 & Ântero-posterior \\
\hline Hyphessobrycon luetkenii & 391 & 29,46 & 0,08 & Ântero-posterior \\
\hline Hyphessobrycon luetkenii & 391 & 31,66 & 0,08 & Ântero-posterior \\
\hline Hyphessobrycon luetkenii & 391 & 31,00 & 0,08 & Ântero-posterior \\
\hline Scinax sp. & 545 & 43,86 & 0,08 & Ântero-posterior \\
\hline Hyphessobrycon luetkenii & 391 & 31,92 & 0,08 & Ântero-posterior \\
\hline Scinax sp. & 545 & 47,50 & 0,09 & Ântero-posterior \\
\hline Odontophrynus americanus & 640 & 58,97 & 0,09 & Ântero-posterior \\
\hline Odontophrynus americanus & 575 & 52,30 & 0,09 & Ântero-posterior \\
\hline Hyphessobrycon luetkenii & 391 & 33,41 & 0,09 & Ântero-posterior \\
\hline Odontophrynus americanus & 342 & 35,19 & 0,10 & Ântero-posterior \\
\hline Hyphessobrycon luetkenii & 391 & 40,06 & 0,10 & Ântero-posterior \\
\hline Oligoryzomys nigripes & 454 & 61,00 & 0,13 & Ântero-posterior \\
\hline Hylidae & 334 & 41,93 & 0,13 & Ântero-posterior \\
\hline Bufo ictericus & 437 & 60,24 & 0,14 & Ântero-posterior \\
\hline Phalloceros caudimaculatus & 245 & 33,80 & 0,14 & Ântero-posterior \\
\hline Oligoryzomys nigripes & 345 & 60,32 & 0,17 & Ântero-posterior \\
\hline Leptodactylus ocellatus & 405 & 68,49 & 0,17 & Ântero-posterior \\
\hline Leptodactylus ocellatus & 451 & 82,12 & 0,18 & Ântero-posterior \\
\hline Scinax fuscovarius & 174 & 35,10 & 0,20 & Ântero-posterior \\
\hline Leptodactylidae & 470 & - & - & Ântero-posterior \\
\hline Anura não identificado & 640 & - & - & Ântero-posterior \\
\hline
\end{tabular}


A correlação entre o tamanho das serpentes (CRC) e o tamanho de suas presas (CT) não foi significativa $(\mathrm{r}=0,0806 ; \mathrm{t}=0,4713 ; \mathrm{P}=$ 0,6404, n = 36) (Figura 1), mas a correlação entre o tamanho da serpente (CT) e a razão tamanho presa / predador (CT / CRC) foi significativa e negativa $(\mathrm{r}=0,6217 ; \mathrm{t}=4,6278 ; \mathrm{P}=$ $0,0001, \mathrm{n}=36$ ) (Figura 2).

\section{Discussão}

A evidente predominância de anfíbios anuros na dieta de Thamnodynastes strigatus permite considerar esta serpente como anurófaga, corrobora as observações de Bernarde $e t$ al. (2000b) e pode indicar que outros grupos de presas, correntemente citados na literatura como itens alimentares da espécie, são de ocorrência ocasional. Embora 28\% dos itens alimentares registrados neste estudo tenham sido peixes, essa categoria de presa pode ser considerada de importância secundária, tendo sido encontrada em apenas três dos 28 espécimes de $T$. strigatus que forneceram dados sobre dieta. De forma análoga, $11 \%(n=3)$ dos itens alimentares registrados para $T$. strigatus por Bernarde et al. (2000b) foram peixes, os quais foram ingeridos por apenas um dos 18 espécimes analisados.

T. strigatus utiliza com freqüência ambientes aquáticos (Franco 1999, Bernarde et al.

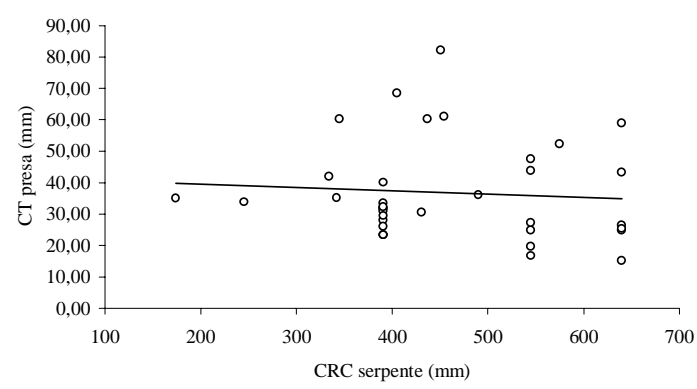

Figura 1 - Relação entre o comprimento rostro-cloacal (CRC) de Thamnodynastes strigatus e o comprimento total (CT) de suas presas, no sul do Brasil. 2000a, b, Giraudo 2001, obs. pess.), corroborando a hipótese segundo a qual a utilização do ambiente por serpentes está relacionada principalmente à distribuição e à abundância de suas presas (Vitt e Vangilder 1983, Vitt 1987, Jennings et al. 1992, Reinert 1993, Bernarde e Kokubum 1999). A freqüente utilização de ambientes aquáticos já foi registrada para outras espécies de Thamnodynastes (Cunha e Nascimento 1978, Lema et al. 1983, Cei 1993, Strüssmann e Sazima 1993, Marques 1998, Franco 1999, Bernarde et al. 2000b, Giraudo 2001), o que pode indicar que essas espécies também baseiem sua dieta em organismos aquáticos.

Entretanto, T. strigatus procura ativamente por presas também em diferentes substratos no solo e sobre a vegetação (Bernarde et al. 2000a, b). Esse comportamento poderia proporcionar o encontro das presas menos usuais registradas em sua dieta (e.g. lagartos, serpentes e roedores) e categorizar T. strigatus como espécie oportunista, mas não generalista, dada a expressiva predominância de anuros em sua dieta. Bernarde $e t$ al. (2000b) também registraram anfíbios anuros como presas predominantes $(71 \%$ dos itens alimentares) de T. strigatus no estado do Paraná, sul do Brasil, e outras categorias de presas em quantidades pouco expressivas (roedores, 14\%; peixes, $11 \%$; lagartos, $4 \%$ ).

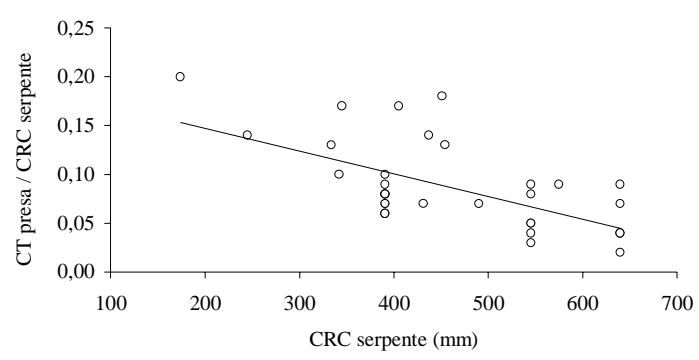

Figura 2 - Relação entre o comprimento rostro-cloacal (CRC) de Thamnodynastes strigatus e a razão comprimento total da presa (CT) / comprimento rostro-cloacal da serpente (CRC), no sul do Brasil. 
Embora muitas espécies de serpentes excluam de sua dieta presas proporcionalmente muito pequenas (Arnold 1993, Greene et al. 1994), outras ingerem presas pequenas com freqüência (Shine 1977, 1987, 1988, Seib 1984, Godley et al. 1984, Greene 1984, Henderson et al. 1987, Slip e Shine 1988, Rowe et al. 2000, Clark 2002, Aguiar e Di-Bernardo, no prelo), como ocorre com T. strigatus. Shine (1977) sugeriu que o custo de captura e ingestão de presas pequenas seria menor em relação ao seu conteúdo energético, quando comparado ao custo de captura e ingestão de presas grandes, e que os riscos de injúria seriam menores. No entanto, a teoria de forrageio prediz que predadores com acesso a presas de variados tamanhos selecionam as presas maiores, maximizando a eficiência do forrageio (Schoener 1971). Ao que parece, T. strigatus alimenta-se indistintamente de presas proporcionalmente grandes ou pequenas, prevalecendo o caráter oportunista condicionado pelo encontro dessas presas.

As presas proporcionalmente maiores foram ingeridas pelas serpentes menores, corroborando a idéia de que o comportamento de capturar presas exageradamente grandes parece ser mais freqüente em indivíduos juvenis de serpentes, seja pela menor disponibilidade de presas de tamanho adequado, seja pela inexperiência e erro de avaliação de serpentes juvenis quanto à sua capacidade de ingestão (Sazima e Martins 1990).

Apenas presas proporcionalmente pequenas foram ingeridas a partir da região posterior do corpo (sentido póstero-anterior), indicando que T. strigatus manipula presas proporcionalmente grandes e as orienta antes da ingestão. Segundo Sazima (1989) e Moori (1991), esse comportamento de ingestão diminui a resistência imposta pelos apêndices locomotores das presas, reduzindo o tempo e a energia despendidos durante a seqüência alimentar.

\section{Agradecimentos}

A Arlei E. Ferreira Velho, Axel Kwet, José
P. da Silva, Mirco Solé e Roberto Baptista de Oliveira, pela identificação de itens alimentares. A Moema Leitão de Araújo, Sônia T. Zanini Cechin e Noeli Zanella, pelo empréstimo de espécimes do MCN, ZUFZM e CRUPF, respectivamente. A Luís Felipe Schmidt de Aguiar, Vanda Lúcia Ferreira, Thales de Lema e Francisco L. Franco pelas críticas e sugestões. Ao CNPq, pelas bolsas de mestrado concedidas a RF e GFM e de Produtividade em Pesquisa concedida a MD.

\section{Referências Bibliográficas}

Ab'Saber, A. N. 1977. Os domínios morfoclimáticos na América do Sul. Primeira aproximação. Geomorfologia 52: 1-21.

Abuys, A. 1986. The snakes of the Surinam, part XV: Subfamily Xenodontinae (Genera Tantilla, Thamnodynastes and Tripanurgos). Litteratura Serpentium 6: 107-116.

Achaval-Elena, F. 2001. Actualización sistemática y mapas de distribuición de los reptiles del Uruguay. Smithsonian Herpetological Information Service 129: 1-37.

Achaval, F. e A. Olmos. 1997. Anfibios y Reptiles del Uruguay. Montevideo. Barreiros y Ramos S.A. 128 pp.

Aguiar, L. F. S. e M. Di-Bernardo. Diet and feeding behavior of Helicops infrataeniatus (Serpentes: Colubridae) in southern Brazil. Studies on Neotropical Fauna and Environment. (no prelo.)

Amaral, A. 1978. Serpentes do Brasil - iconografia colorida. $2^{a}$ ed. São Paulo. Melhoramentos. 248 pp.

Arnold, S. J. 1993. Foraging theory and prey-size-predatorsize relations in snakes. Pp. 87-115 in R. A. Seigel e J. T. Collins (eds.), Snakes - ecology and behavior. New York. McGraw-Hill.

Bailey, J. R. 1967. The synthetic approach to colubrid classification. Herpetologica 23: 155-161.

Bernarde, P. S. e M. N. C. Kokubum. 1999. Anurofauna do município de Guararapes, Estado de São Paulo, Brasil (Amphibia: Anura). Acta Biológica Leopoldensia 21: 88-97.

Bernarde, P. S., M. N. C. Kokubum e O. A. V. Marques. 2000a. Utilização de hábitat e atividade em Thamnodynastes strigatus (Günther, 1858) no sul do Brasil (Serpentes, Colubridae). Boletim do Museu Nacional 428: 1-8.

Bernarde, P. S., J. C. Moura-Leite, R. A. Machado e M. 
N. C. Kokubum. 2000b. Diet of the colubrid snake Thamnodynastes strigatus (Günther, 1858) from Paraná State, Brazil, with field notes on anuran predation. Revista Brasileira de Biologia 60: 695-699.

Bizerra, A. F. 1998. História natural de Tomodon dorsatus (Serpentes, Colubridae). Dissertação de Mestrado Nãopublicada. Universidade de São Paulo. Brasil.

Cechin, S. T. Z. 1999. História natural de uma comunidade de serpentes na região da Depressão Central (Santa Maria), Rio Grande do Sul, Brasil. Tese de Doutorado Não-publicada. Pontifícia Universidade Católica do Rio Grande do Sul. Brasil.

Cei, J. 1993. Reptiles del Noroeste y Este de la Argentina. Herpetofauna de las Selvas Subtropicales, Puna y Pampas. Torino. Museo Regionale di Scienze Naturali (Monografie 14). 949 pp.

Clark, R. W. 2002. Diet of the Timber Rattlesnake, Crotalus horridus. Journal of Herpetoloy 36: 494499.

Cunha, O. R. e F. P. Nascimento. 1978. Ofídios da Amazônia X - As cobras da região leste do Pará, Belém. Publicações Avulsas do Museu Paraense Emílio Goeldi 31: 1-218.

Franco, F. L. 1999. Relações filogenéticas entre os gêneros da tribo Tachymenini Bailey (1967) (Serpentes; Colubridae). Tese de Doutorado Não-publicada. Universidade de São Paulo. Brasil.

Franco, F. L. e T. G. Ferreira. 2002. Descrição de uma nova espécie de Thamnodynastes Wagler, 1830 (Serpentes, Colubridae) do nordeste brasileiro, com comentários sobre o gênero. Phyllomedusa 1: 57-74

Giraudo, A. R. 2001. Serpientes de la Selva Paranaense $y$ del Chaco Húmedo. Buenos Aires. Literature of Latin America. 328 pp.

Godley, J. S., R. W. McDiarmid e N. N. Rojas. 1984. Estimating prey size and number in crayfish-eating snakes, genus Regina. Herpetologica 40: 82-88.

Greene, H. W. 1984. Feeding behavior and diet of the eastern coral snake, Micrurus fulvius. Pp.147-162 in R. A. Seigel, L. E.Hunt, J. L. Knight, L. Malaret e N. L. Zuschlag (eds.), Vertebrate Ecology and Systematics - a tribute to Henry S. Fitch. Special Publication 10. Lawrence. Museum of Natural History.

Greene, B. D., J. R. Dixon, J. M. Mueller, M. J. Whiting e O. W. Thornton-Jr. 1994. Feeding ecology of the Concho Water Snake, Nerodia harteri paucimaculata. Journal of Herpetology 28: 165-172.

Günther, A. 1858. Catalogue of Colubrine snakes in the collection of the British Museum. London. Trustees of the British Museum. 281pp.

Henderson, R. W., A. Schwartz e T. A. Noeske-Halin. 1987. Food habits of three colubrid tree snakes (genus
Uromacer) on Hispaniola. Herpetologica 43: 241-248.

Jennings, W. B., D. F. Bradford e D. F. Johnson. 1992. Dependence of the garter snake Thamnophis elegans on amphibians in the Sierra Nevada of California. Journal of Herpetology 26: 503-505.

Lema, T. 2002. Os Répteis do Rio Grande do Sul - atuais e fósseis, biogeografia, ofidismo. Porto Alegre. EDIPUCRS. 264 pp.

Lema, T., M. Leitão-de-Araújo e A. C. P. Azevedo. 1983. Contribuição ao conhecimento da alimentação e do modo alimentar de serpentes do Brasil. Comunicações do Museu de Ciências da PUCRS (série Zoologia) 26: 41-121.

Lema, T., M. I. Vieira e M. Leitão-de-Araújo. 1984. Fauna reptiliana do norte da Grande Porto Alegre, Rio Grande do Sul, Brasil. Revista Brasileira de Zoologia 2: 203-227.

Marques, O. 1998. Composição faunística, história natural e ecologia de serpentes da Mata Atlântica na região da Estação Ecológica Juréia-Itatins. Tese de Doutorado Não-publicada. Universidade de São Paulo. Brasil.

Marques, O. A. V., A. Eterovic e I. Sazima. 2001. Serpentes da Mata Atlântica - guia ilustrado para a Serra do Mar. Ribeirão Preto. Holos. 184 pp.

Moori, A. 1991. Effects of prey size and type on prey handling behavior in Elaphe quadrivirgata. Journal of Herpetology 25: 160-166.

Peters, J. A. e B. Orejas-Miranda. 1970. Catalogue of the Neotropical Squamata. Part I. Snakes. Bulletin of the United States National Museum 297: 1-347.

Reinert, H. K. 1993. Habitat selection in snakes. Pp. 201240 in R. A. Seigel e J. T. Collins (eds.), Snakes ecology and behavior. New York. McGraw-Hill.

Rowe, J. W., K. C. Campbell e J. C. Gillingham. 2000. Diet of the ribbon snake on Beaver Island, Michigan: temporal variation and the relationship of prey size to predator size. Herpetological Natural History 7: 145152.

Sazima, I. 1989. Comportamento alimentar da jararaca, Bothrops jararaca: encontros provocados na natureza. Ciência e Cultura 41: 500-505.

Sazima, I. e M. Martins. 1990. Presas grandes e serpentes jovens: quando os olhos são maiores que a boca. $M e$ mórias do Instituto Butantan 52: 73-79.

Schoener, T. W. 1971. Theory of feeding strategies. Annual Review of Ecology and Systematics 11: 69-404.

Seib, R. L. 1984. Prey use in three syntopic neotropical racers. Journal of Herpetology 18: 412-420.

Slip, D. J. e R. Shine. 1988. Feeding habits of the diamond python, Morelia s. spilota: ambush predation by a boid snake. Journal of Herpetology 22: 323-330. 
Shine, R. 1977. Habitats, diets and sympatry in snakes: a study from Australia. Canadian Journal of Zoology 55: $1118-1128$.

Shine, R. 1987. Ecological ramifications of prey size: food habits and reproductive biology of Australian copperhead snakes (Austrelaps, Elapidae). Journal of Herpetology 21: 21-28.

Shine, R. 1988. Food habits and reproductive biology of small Australian snakes of the genera Unechis and Suta (Elapidae). Journal of Herpetology 22: 307-315.

Strüssmann, C. e I. Sazima. 1993. The snake assemblage of the Pantanal at Poconé, western Brazil: faunal composition and ecological summary. Studies on Neotropical Fauna and Environment 28: 157-168.

Vanzolini, P., A. Ramos-Costa e L. J. Vitt. 1980. Répteis das Caatingas. Rio de Janeiro. Academia Brasileira de Ciências. 161pp.

Vitt, L. J. 1987. Communities. Pp. 335-365 in R. A. Seigel, J. T. Collins e S. S. Novak (eds.), Snakes - ecology and evolutionary biology. New York. MacGraw-Hill.

Vitt, L. J. e L. D. Vangilder. 1983. Ecology of a snake community in northeastern Brazil. Amphibia-Reptilia 4: 273-296. 